Collection SFN 10 (2010) 545-562

(C) Owned by the authors, published by EDP Sciences, 2010

DOI: $10.1051 / \mathrm{sfn} / 2010013$

\title{
Les clathrates hydrates
}

\author{
A. Desmedt
}

ISM - Groupe de Spectroscopie Moléculaire, UMR 5255 CNRS, Université de Bordeaux 1, 351 cours de la Libération, 33405 Talence Cedex, France

e-mail : a.desmedt@ism.u-bordeaux1.fr

\section{INTRODUCTION}

Les clathrates hydrates sont des cristaux organiques nanoporeux dans lesquels les molécules d'eau forment des cages pouvant encapsuler un grand nombre d'espèces moléculaires, la topologie des cages aqueuses dépendant de la nature des molécules invitées [1]. La structure cristalline des clathrates hydrates (Figure 1) peut être décrite sur la base de polygones plans, formés par des molécules d'eau en liaisons hydrogène. L'angle $\mathrm{H}-\mathrm{O}-\mathrm{H}$ étant de $109^{\circ}$, $1^{\prime}$ arrangement le plus stable est de type pentagonal. En utilisant ce motif élémentaire, il est possible de former des cages dodécaédriques ayant un diamètre de $7.8 \AA$ (dénotées $5^{12}$ en référence aux 12 pentagones nécessaires pour la former). Bien qu'énergétiquement défavorables, des hexagones ou des carrés peuvent conduire à la formation de cages pouvant accommoder des molécules invitées «volumineuses ». Par exemple, l'association de 12 pentagones et de 2 ou 4 hexagones donne naissance à des cages de types $5^{12} 6^{2}$ (diamètre de $8.7 \AA$ ) ou $5^{12} 6^{4}$ (diamètre de $9.5 \AA$ ), respectivement. Trois des quatre axes de coordinations de l'oxygène sont utilisés pour former ces cages, de sorte que le quatrième est disponible pour former un cristal tridimensionnel. La combinaison de ces différents types de cage conduit alors à plusieurs structures cristallines, dont les plus communément rencontrées sont les types I, II et H représentés sur la Figure 1. Le réseau de liaison hydrogène entre molécules d'eau présente un désordre d'origine dynamique, respectant la règle de la glace (c'est-à-dire que chaque molécule d'eau accepte et donne 2 liaisons hydrogène). En effet, des études structurales ont montrées que les atomes d'hydrogène occupent les deux sites possibles dans chaque liaison $\mathrm{O}-\mathrm{H}^{\cdots} \mathrm{O}$ avec une probabilité de 1/2 [2-4]. Enfin, il est intéressant de noter que pour un remplissage total des cages d'un hydrate, les trois structures rencontrées (Figure 1) sont constituées à $85 \%$ de molécules d'eau et à $15 \%$ de molécules invitées. Ce remplissage optimal des cages n'est pas systématique. Suivant la molécule invitée et le rapport molaire invité/eau utilisé (pour la cristallogenèse en laboratoire de clathrate hydrate), seul un type de cage peut être rempli et le plus souvent, les différents types de cages ont des probabilités d'occupation différentes (on parle alors de clathrate non-stoichiométrique).

Les recherches entreprises sur les clathrates hydrates sont actuellement en plein essor en raison de la diversité des systèmes dans lesquels ils sont présents (fonds des océans, planètes, comètes, intérieur des cellules au voisinage de biomolécules hydrophobes...) et de leurs propriétés physicochimiques spécifiques touchant plusieurs disciplines (environnement, énergie...) [5-8]. Parmi les nombreuses variétés de clathrates existants, les hydrates de gaz représentent certainement les systèmes les plus étudiés (l'hydrate de méthane étant le plus connu). A l'état naturel, de grandes quantités d'hydrate de gaz se trouvent dans les fonds océaniques ou le permafrost, milieux où les conditions particulières de température et de pression sont favorables à leur stabilité. Ces réserves représentent un potentiel énergétique non exploité, probablement supérieur à la totalité des ressources connues de combustibles fossiles. D’un point de vue environnemental, ces réserves pourraient avoir un impact

This is an Open Access article distributed under the terms of the Creative Commons Attribution-Noncommercial License 3.0, which permits unrestricted use, distribution, and reproduction in any noncommercial medium, provided the original work is properly cited. 
sur les changements climatiques globaux (le méthane étant un gaz à effet de serre). Pour l'industrie pétrolière, ces hydrates de gaz constituent des pertes économiques importantes du fait de leur formation dans les pipelines (réduisant ou obstruant le flux des hydrocarbures) ou encore du fait des dangers d'explosion ou d'incendie lors des forages (instabilité au niveau des couches d'hydrate de gaz libérant des poches de gaz). De plus, ces systèmes ouvrent de nouvelles perspectives industrielles pour le stockage et le transport de gaz naturel dans des conditions douces (les hydrates de gaz sont stables à pression atmosphérique aux alentours de $250 \mathrm{~K}$ alors, que le gaz naturel liquéfié doit être maintenu à $110 \mathrm{~K}$ ) [7]. Enfin, des travaux récents ont montré que les clathrates hydrates pouvaient également être utilisés pour le stockage d'hydrogène [9-11].

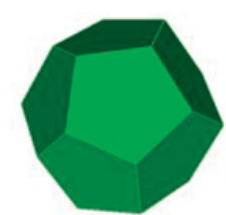

Type de eage $\quad 5^{12}$

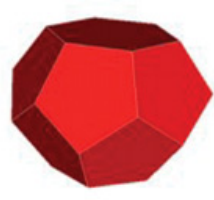

$5^{12} 6^{2}$

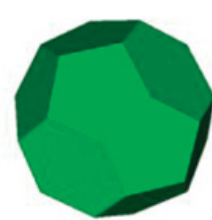

$5^{12} 6^{4}$

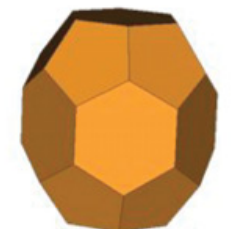

$5^{12} 6^{8}$

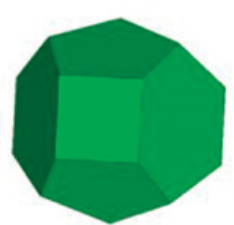

$4^{3} 5^{6} 6^{3}$

Diamètre moyen $7.82 \AA$ $8.66 \AA$

Nombre de cages par maille élémentaire

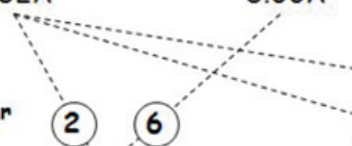

Type I

Cubique, $a \approx 12 \AA$ Pm3n-46 $\mathrm{H}_{2} \mathrm{O}$ par maille

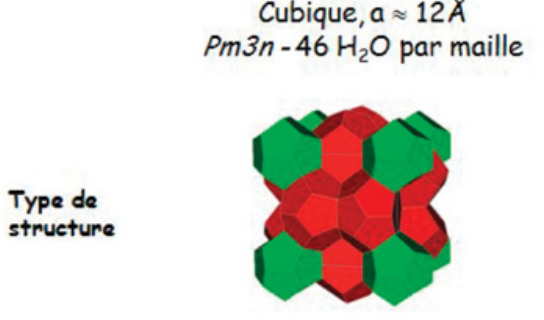

$9.46 \AA$
$11.42 \AA$
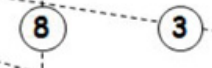

(16.)

Type II

Cubique, $a \approx 17 \AA$ Fd3m-136 $\mathrm{H}_{2} \mathrm{O}$ par maille

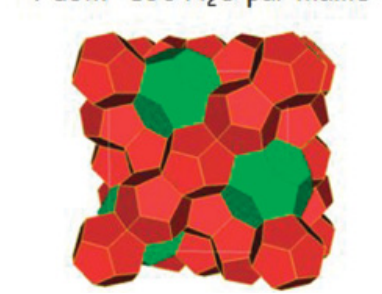

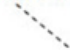

1

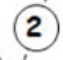

Type $\mathrm{H}$

Héxagonale, $a \approx 12 \AA, c \approx 10 \AA$ $\mathrm{P} 6 / \mathrm{mmm}-34 \mathrm{H}_{2} \mathrm{O}$ par maille

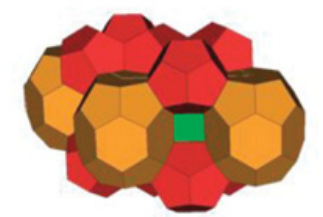

Figure 1. Représentation des cages et des structures cristallines des clathrates hydrates les plus communes. Les polyèdres sont formés à partir de la position moyenne des atomes d'oxygène des molécules d'eau et les arêtes contiennent les liaisons hydrogène entre molécules d'eau.

Les études menées actuellement visent à comprendre à un niveau fondamental les propriétés physico-chimiques spécifiques aux clathrates hydrates. De nombreuses publications sont consacrées aux facteurs régissant la formation, l'inhibition et la décomposition des hydrates de gaz, aux propriétés thermodynamiques et d'équilibre de phase des hydrates et à la conductivité thermique "anormale" de ces systèmes. L'étude des propriétés structurales et dynamiques des clathrates hydrates joue un rôle prépondérant dans la compréhension à un niveau microscopique de ces propriétés macroscopiques [12]. L'objectif de ce cours est d'illustrer l'apport de la diffusion inélastique des neutrons dans l'étude des propriétés dynamiques des clathrates hydrates ${ }^{1}$. En particulier, bien que les interactions hôte-invité dans les clathrates hydrates «ne soient que »de type van der Waals, leur nature relativement forte

\footnotetext{
${ }^{1}$ Le formalisme associé au calcul des différentes grandeurs physiques (loi de diffusion, densité d'état, etc...) à partir des spectres expérimentaux ne sera pas décrit dans ce cours. Le lecteur pourra se reporter sur les articles parus dans le même volume [13] ou dans les volumes précédents $[14,15]$.
} 
pilote les propriétés spécifiques de ces systèmes et cette propriété sera soulignée à travers les exemples choisis. Dans cette perspective, les caractéristiques principales des excitations rencontrées dans ces systèmes seront examinées en premier partie. Les résultats présentés s'appuient sur des résultats de diffusion inélastique des neutrons, mais également sur des techniques complémentaires telles que la spectroscopie Raman. Dans ces études, il faut également souligner le développement des approches combinant expérience et théorie. En particulier, les approches théoriques à l'aide des simulations de dynamique moléculaire ou de dynamique de réseau [16] permettent de guider l'interprétation des résultats expérimentaux tout en apportant des informations originales. La complémentarité de la diffusion neutronique et des simulations de dynamique moléculaire réside dans les observables communes (l'évolution dans le temps de la position d'atomes) rendant les comparaisons totalement naturelles. Dans une seconde partie, l'importance des couplages hôte-invité dans ces systèmes sera exposée à travers la présentation de deux propriétés remarquables des clathrates hydrates : leur conductivité thermique « anormale » et l'existence de site d'adsorption à la surface des cages.

\section{GÉNÉRALITÉS SUR LA DYNAMIQUE DES CLATHRATES HYDRATES}

Afin de fixer les idées quant à la problématique de la diffusion inélastique (des neutrons) dans les clathrates hydrates, il est utile de rappeler quelques points concernant la dynamique individuelle (hors vibrations intramoléculaires) des molécules encagées et des molécules d'eau, c'est-à-dire d'évoquer la diffusion «quasi-(in)élastique ». Les deux entités moléculaires constituant les clathrates hydrates présentent un fort désordre dynamique sur des échelles de temps différentes. Typiquement, la molécule incluse se réorientera avec un temps caractéristique de l'ordre de la picoseconde (par exemple, voir les études menées par RMN sur le clathrate hydrate modèle formé à partir du tétrahydrofuranne [17-19]). Les molécules d'eau ont des temps de réorientation de l'ordre de la microseconde [2]. Cette échelle de temps est valable pour des clathrates hydrates «standard » (c'est-à-dire ne présentant que très peu de défaut au niveau du réseau de liaisons hydrogène de la sous-structure aqueuse). Dans le cas de clathrate hydrate d'acide, le confinement d'acides dans les cages d'un clathrate hydrate va générer des excès de proton délocalisés (c'est-à-dire des défauts de liaisons hydrogène au niveau de la sousstructure aqueuse). Ces clathrates hydrates ioniques (très bons conducteurs de proton) présentent alors des réorientations de molécules d'eau sur une échelle de temps de l'ordre de la nanoseconde [20].

Les interactions hôte-invité dans ces systèmes se feront alors sur des échelles de temps plus courtes, directement accessibles par diffusion inélastique des neutrons. Dans cette section, nous allons présenter les principales caractéristiques des excitations rencontrées dans les clathrates hydrates en allant des modes de vibrations intramoléculaires aux modes de basse fréquence. Outre leur intérêt fondamental, de telles études permettent de déterminer des signatures spectroscopiques d'un clathrate hydrate ayant de nombreuses implications telles que la détection de clathrates hydrates en astrophysique ou la cartographie in-situ de la dispersion d'hydrate de gaz dans les fonds océaniques.

\subsection{Modes intramoléculaires}

Outre la diffusion inélastique incohérente des neutrons, les techniques de diffusion Raman permettent de sonder les modes de vibration intramoléculaire à des nombres d'onde allant d'une centaine à quelques milliers de $\mathrm{cm}^{-1}$ [21]. Etant aisément mise en ouvre dans les laboratoires et permettant des études in-situ (à condition de disposer de cellule optique permettant de travailler en température et pression), cette technique spectroscopique est principalement utilisée pour ce type d'analyse. Dans ce paragraphe, nous allons illustrer le fait que ces études fournissent de précieuses informations sur les interactions hôtes-invités ou sur les mécanismes de formation des clathrates hydrates.

Les spectres Raman correspondant aux élongations $\mathrm{CH}$ du méthane dans les structures de type I et type II sont présentés sur la Figure 2. A l'état gazeux, une seule bande d'élongation symétrique $\mathrm{CH}$ du méthane est observée en Raman (à $2917 \mathrm{~cm}^{-1}$ ). Les deux bandes observées dans le cas des clathrates 
hydrates sont ainsi attribuées au méthane occupant les deux types de cages des structures I ou II. De plus, les rapports d'intensité des bandes (après déconvolution) sont fortement corrélés au rapport du nombre de cages occupées dans chacune des structures. Sur la base de ces observations, il est possible d'effectuer l'attribution suivante (voir les Figure 1 et Figure 2) :

- Type I. Sachant que la bande à $2904 \mathrm{~cm}^{-1}$ est trois fois plus intense que celle à $2915 \mathrm{~cm}^{-1}$ et $\overline{\text { qu'il y }}$ a trois fois plus de cages de type $5^{12} 6^{2}$ que de cages de type $5^{12}$, la bande à $2904 \mathrm{~cm}^{-1}$ est attribuée aux élongations des molécules de méthane dans les grandes cages et celle à $2915 \mathrm{~cm}^{-1}$ aux petites cages.

- Type II : De manière analogue, la bande à $2905 \mathrm{~cm}^{-1}$ est attribuée au méthane dans les grandes cages (de type $5^{12} 6^{4}$ ) et celle à $2910 \mathrm{~cm}^{-1}$, au méthane dans les petites cages (de type $5^{12}$ ).

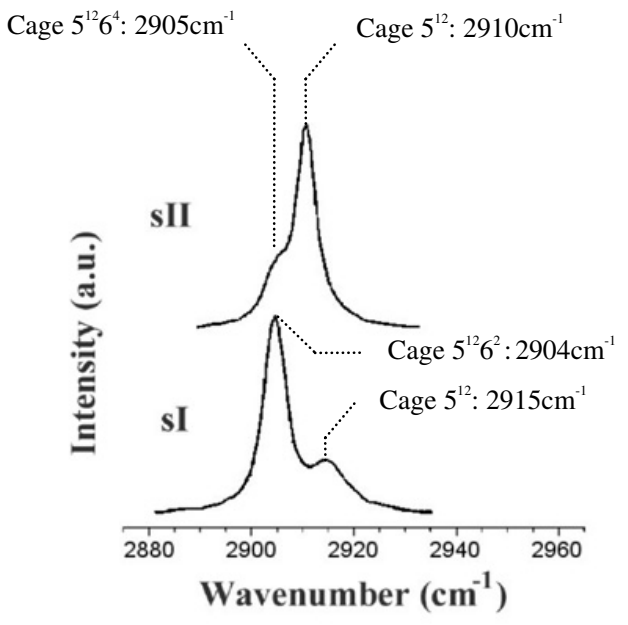

Figure 2. Spectres de diffusion Raman des élongations $v(\mathrm{CH})$ du méthane dans les structure de type II et de type I ( $\mathrm{T}=298 \mathrm{~K}$ et $\mathrm{P}=1250$ bars). Adapté de [22].

D'un point de vue fondamental, il est intéressant de souligner le fait que la fréquence d'élongation du méthane à l'état gazeux (notée $v_{\text {gaz }}$ ) est plus haute que celles du méthane contenu dans les cages d'un clathrate hydrate. Par contre, que ce soit pour la structure de type I ou pour celle de type II, les élongations sont observées à plus basses fréquences dans les grandes cages (notée $v_{\mathrm{GC}}$ ) que dans les petites cages (notée $\nu_{\mathrm{PC}}$ ). Ces observations peuvent paraître contradictoires. En effet, en considérant le cas du gaz comme un «confinement dans une cage de dimension infinie », on s'attendrait à avoir $v_{\mathrm{gaz}}>v_{\mathrm{GC}}>v_{\mathrm{PC}}$, alors qu'expérimentalement, on observe $v_{\mathrm{gaz}}>v_{\mathrm{PC}}>v_{\mathrm{GC}}$. Ces observations peuvent toutefois être rationalisées à l'aide du modèle «loose-cage tight-cage » développé par Buckingham, Pimentel et Charles (voir les références citées dans [23]). Ce modèle permet de quantifier la différence des fréquences d'élongation d'une molécule diatomique à l'état gazeux et en milieu confiné à partir des dérivées premières et secondes du potentiel d'interaction entre la molécule diatomique et son environnement. En appliquant ce modèle au cas du clathrate hydrate de méthane et en supposant une interaction attractive entre le méthane et la cage aqueuse [23], la fréquence du mode d'élongation du méthane confiné dans la cage d'un clathrate hydrate est effectivement plus basse que celle à l'état gazeux. De plus, du fait d'une distance méthane-cage plus grande dans les grandes cages que dans les petites cages et d'un allongement de la liaison C-H dans les grandes cages [24], il est bien attendu que $\nu_{\mathrm{PC}}>v_{\mathrm{GC}}$. Ces résultats supposent ainsi l'existence de «fortes interactions » entre les molécules incluses et la sous-structure aqueuse comme cela sera illustré dans la section 3. 
Le couplage d'un spectromètre Raman avec un microscope (confocal) permet l'étude d'échantillon de taille micrométrique, aspect particulièrement attractif pour des études in-situ des clathrates hydrates (détection dans les milieux naturels par exemple). Un exemple d'application concerne le suivi de la cinétique de formation des clathrates hydrates par diffusion Raman [25,26]. Sur la Figure 3, l'évolution en temps réel des bandes d'élongations $v(\mathrm{CH})$ du méthane est représentée lors de la formation de l'hydrate de méthane (spectre à $t=12000 \mathrm{~s}$ ) à partir d'une solution méthane-eau sous une pression de 317 bars (spectre à $t=0 \mathrm{~s}$ ). La bande à $2911 \mathrm{~cm}^{-1}$ associée au méthane dissout dans l'eau se sépare en deux bandes distinctes et caractéristiques de la structure de type I du clathrate hydrate de méthane. Il est possible de déconvoluer ces deux bandes pour déterminer les occupations relatives de chaque type de cage [25]. Ce type d'approche apporte des informations cruciales permettant de vérifier expérimentalement les mécanismes de formation proposés pour ces hydrates [27] : des cages labiles de type $5^{12}$ sont initialement formées et la transformation de ces cages en cages de type $5^{12} 6^{2}$ est le facteur limitant lors de la formation de la structure de type I.

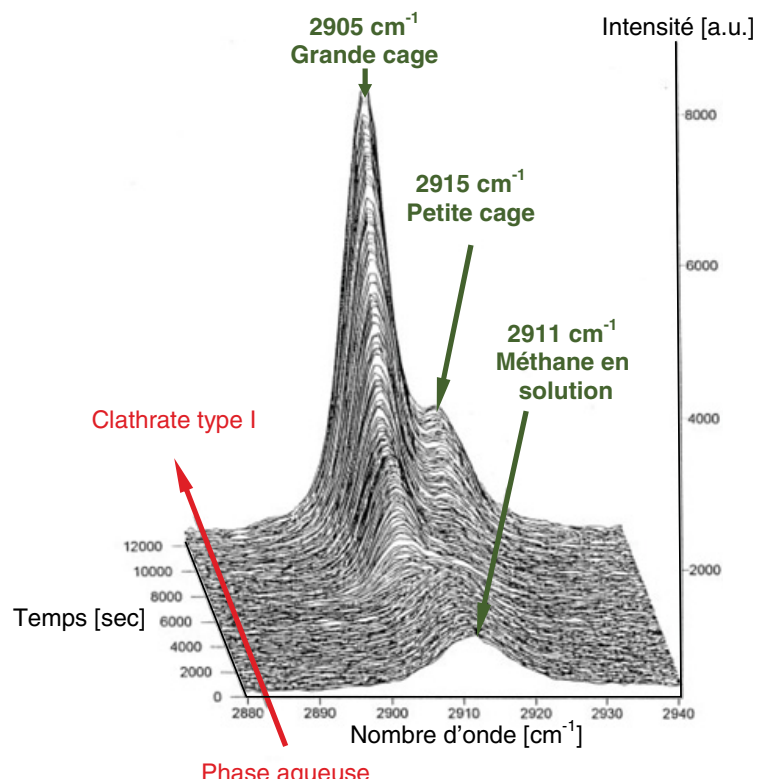

Figure 3. Spectres de diffusion Raman ( $25 \mathrm{~s}$ par spectre) enregistrés durant la formation de l'hydrate de méthane à $\mathrm{P}=317$ bars. $t=0 \mathrm{~s}$ : méthane dissous dans de l'eau à $\mathrm{T}=297.15 \mathrm{~K} . t=12000 \mathrm{~s}$ : hydrate de méthane à $\mathrm{T}=275.65 \mathrm{~K}$. Vitesse de refroidissement : $0.1 \mathrm{~K} \cdot \mathrm{min}^{-1}$. Adapté de [25].

\subsection{Modes de basse fréquence}

La spectroscopie de diffusion inélastique des neutrons est particulièrement utilisée pour l'étude des modes de basses fréquences dans les clathrates hydrates. Toutefois, les études menées sont basées sur des mesures de densités d'état étant donnée la difficulté pour obtenir des mono-cristaux de taille suffisante pour la diffusion neutronique. Cette projection des modes de réseau sur l'axe des transferts d'énergie entraîne la perte de l'information géométrique (c'est-à-dire la dépendance en vecteur d'onde). L'analyse des données fait alors appel à des substitutions isotopiques (principalement, $\mathrm{H}_{2} \mathrm{O} \leftrightarrow \mathrm{D}_{2} \mathrm{O}$ ), à des comparaisons entre clathrates hydrates de même structure mais encapsulant différentes molécules pour analyser ces données, ou encore à des approches par simulations numériques. 


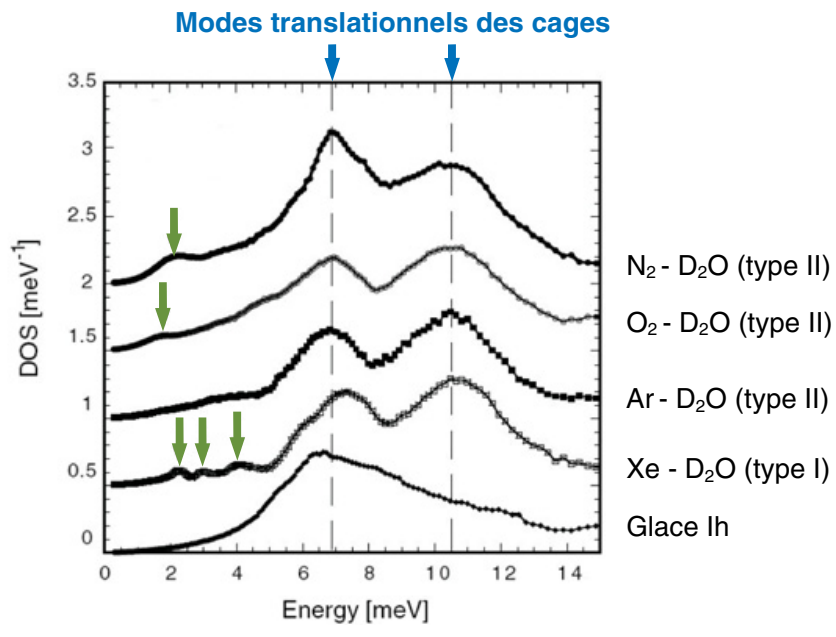

Figure 4. Densité d'état généralisée de différents clathrates hydrates $\left(\mathrm{T}=120 \mathrm{~K}\right.$ sauf pour $\left.\mathrm{O}_{2}, \mathrm{~T}=100 \mathrm{~K}\right)$ et de la glace hexagonale $(\mathrm{T}=235 \mathrm{~K})$. Tiré de [23].

A partir de spectres de diffusion inélastique des neutrons, il est possible de calculer les densités d'état généralisées (notées DOS) ${ }^{2}$, présentées sur la Figure 4 pour différents clathrates hydrates ayant des matrices aqueuses deutérées [23-28]. Les modes observés à 7 et $10.5 \mathrm{meV}$ sont attribués aux mouvements de translation de la sous-structure aqueuse [29]. On peut remarquer un épaulement à basse fréquence de la bande à $7 \mathrm{meV}$ du clathrate hydrate de xénon, signature caractéristique de la structure de type I. Les modes en-dessous de $5 \mathrm{meV}$ sont attribués aux molécules encapsulées. Pour effectuer cette analyse dans le cas des structures de type II, il est judicieux de s'appuyer sur le fait que la section efficace de diffusion de l'argon $(\sigma=0.7$ barn) est négligeable en comparaison avec celle de l'oxygène ( $\sigma=4.2$ barn) ou de l'azote ( $\sigma=11.5$ barn). En supposant que les spectres dus à la sous-structure aqueuse sont identiques pour tous les clathrates hydrates de type II, il est possible d'extraire la contribution des molécules invitées à la densité d'état généralisée par différentiation des DOS des clathrates hydrates de $\mathrm{O}_{2}$ ou de $\mathrm{N}_{2}$ avec la DOS du clathrates hydrates d'argon. Le résultat ainsi obtenu est présenté sur la Figure 5. Les deux bandes observées pour chacun des clathrates hydrates sont attribuées aux molécules invitées dans les deux types de cages de la structure de type II. Il est important de noter qu'en se plaçant dans l'hypothèse harmonique, la différence de masse ne suffit pas à expliquer le ramollissement des modes des molécules d'oxygène par rapport à ceux des molécules d'azote (voir partie gauche de la Figure 5). La différence observée ne peut être due qu'à des différences d'interaction hôte-invité. De plus, en s'appuyant sur des résultats de simulations de dynamique moléculaire [23] (voir la partie droite de la Figure 5), la bande de plus basse fréquence est due aux molécules encapsulées dans les grandes cages. Ainsi, comme cela a été souligné dans la description des spectres de vibrations intramoléculaires, ces résultats mettent à nouveau en évidence l'importance des couplages hôte-invité dans les clathrates hydrates.

Les modes de basse fréquence associés aux molécules encapsulées dans les cages d'un clathrate hydrate sont de type «hochet $»^{3}$. Nous allons maintenant détailler un exemple illustrant l'apport des simulations de dynamique moléculaire (noté MD) dans l'interprétation de ces modes de basse fréquence [31]. Le clathrate hydrate de iodométhane cristallise dans une structure de type II dans laquelle seules les grandes cages (de type $5^{12} 6^{4}$ ) sont remplies par le iodométhane. Sur le spectre de diffusion

\footnotetext{
${ }^{2}$ Les détails expérimentaux concernant ces densités d'état peuvent être trouvés dans les références [23,28].

${ }^{3}$ En anglais, « rattling mode ».
} 

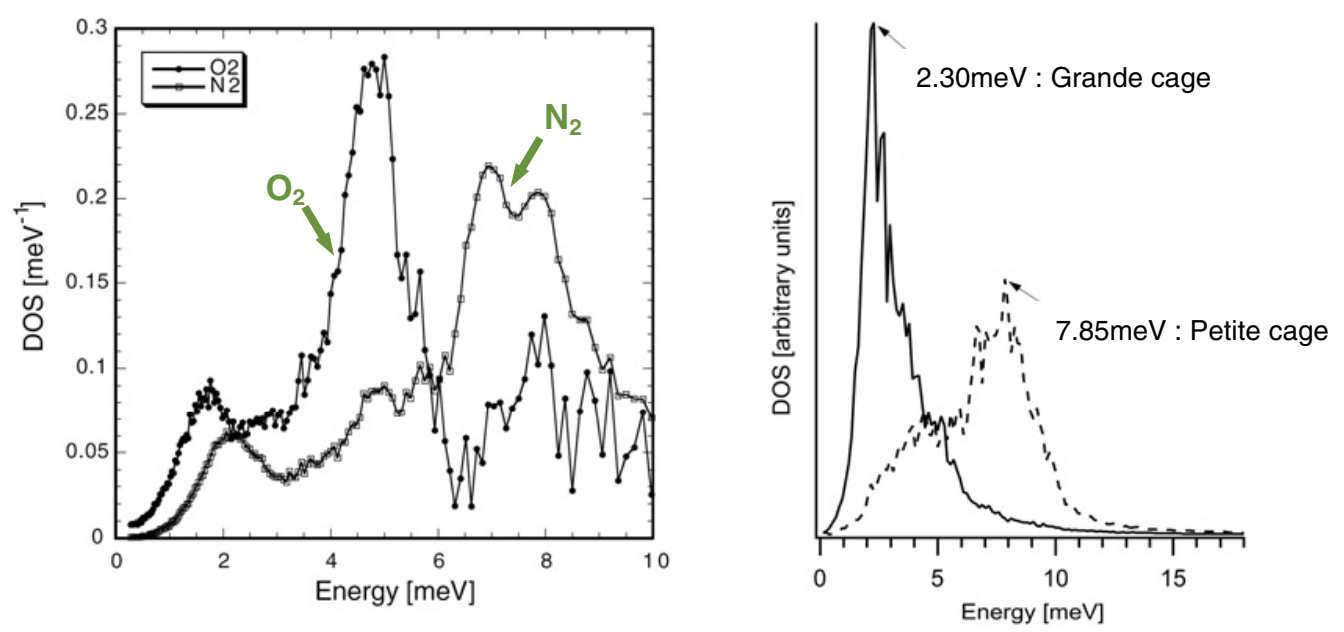

Figure 5. Contribution des molécules invitées aux densités d'état généralisées. A gauche : résultats expérimentaux pour les clathrates hydrates de $\mathrm{N}_{2}(\mathrm{~T}=120 \mathrm{~K})$ et de $\mathrm{O}_{2}(\mathrm{~T}=100 \mathrm{~K})$. A droite : résultat théorique issu de simulation de dynamique moléculaire sur le clathrate hydrate de $\mathrm{N}_{2}(\mathrm{~T}=80 \mathrm{~K})$. Tiré de [23].

quasi-élastique des neutrons de cet échantillon (Figure 6), un épaulement vers $1 \mathrm{meV}$ peut-être observé (celui-ci est mis en évidence en calculant la susceptibilité ${ }^{4}$ ). Dans une analyse expérimentale, il est relativement difficile de déconvoluer ce pic de l'élargissement quasi-élastique. A partir des trajectoires de simulations de dynamique moléculaire, il est possible de calculer les lois de diffusion neutronique et de comparer celles-ci avec les lois expérimentales. Cette comparaison conduit à un très bon accord (Figure 6) et permet ainsi de valider les MD. Il est alors possible de «décortiquer les trajectoires $\mathrm{MD} »$ afin de faciliter l'interprétation de ce mode de basse fréquence. En particulier, il est possible de calculer la loi de diffusion intermédiaire à partir de la trajectoire du centre de masse des molécules de iodométhane (Figure 6). Cette loi peut être ajustée à l'aide d'un modèle d'oscillateur harmonique amorti (ligne continue sur la partie droite de la Figure 6). Ce mode translationnel amorti (observé à $0.95 \mathrm{meV}$ sur les spectres expérimentaux et calculés à partir des MD) est ainsi attribué au «rattling 》 de la molécule encapsulée dans sa cage.

Il faut souligner que ces modes présentent un caractère faiblement anharmonique, du à la nature plane des puits de potentiel des cages aqueuses. Expérimentalement, cette anharmonicité est relativement difficile à mettre en évidence, car ses effets sont couplés à ceux dus aux interactions hôteinvité [23]. Le lecteur pourra se référer aux articles $[23,28]$ pour le cas spécifique des clathrates hydrates et à l'article [30] pour une description plus générale sur les modes « hochet ».

\section{ILLUSTRATIONS DU COUPLAGE HÔTE-INVITÉ DANS LES CLATHRATES HYDRATES}

Les clathrates hydrates sont souvent décrits comme des systèmes dans lesquels les interactions entre les sous-structures hôte et invitée sont faibles, c'est-à-dire de type van der Waals. Or, de nombreuses études ont démontré l'existence de «fortes » interactions entre les deux sous-structures. Afin d'illustrer l'apport de la diffusion inélastique des neutrons à l'étude des couplages hôte-invité des clathrates hydrates, deux exemples ont été sélectionnés. Le premier concerne les propriétés thermiques de ces systèmes. Bien que

\footnotetext{
${ }^{4}$ La susceptibilité est donnée par la relation $S(Q, \omega)=1 / \pi[1+n(\omega)] \chi^{\prime \prime}(\omega)$ où $S(Q, \omega)$ est la loi de diffusion et $n(\omega)$ est le facteur de Bose-Einstein.
} 

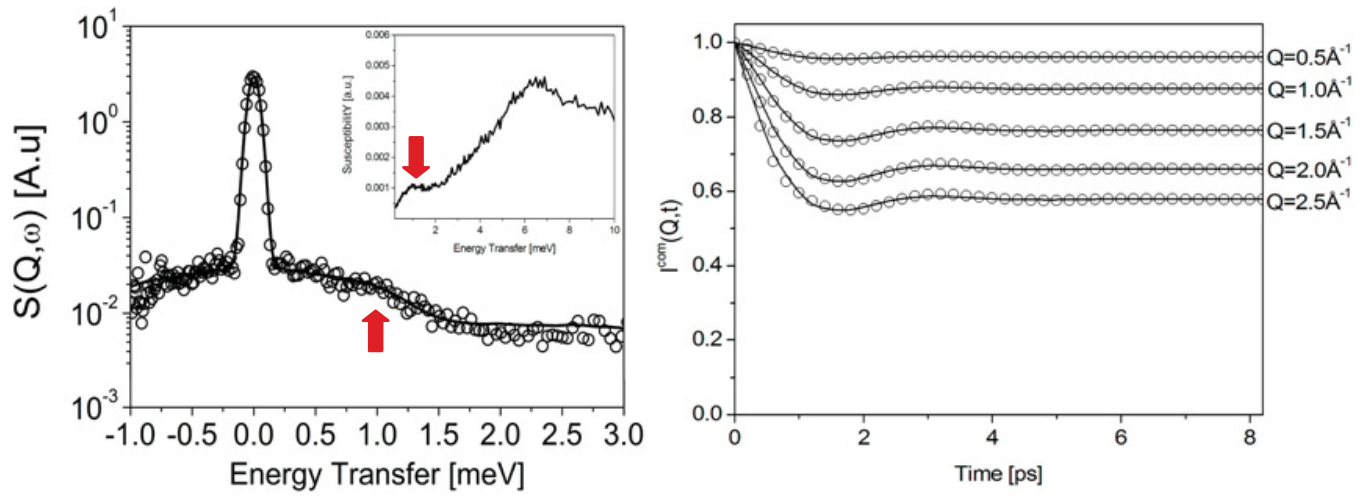

Figure 6. A gauche : Spectre quasi-élastique des neutrons (points) et susceptibilité (en insert) enregistrés sur le clathrate hydrate de iodo-méthane à $\mathrm{T}=150 \mathrm{~K}$ et $\mathrm{Q}=1.4 \AA^{-1}$ avec le spectromètre temps-de-vol NEAT (HZB, Berlin). La ligne continue correspond aux spectres quasi-élastique calculés à partir des trajectoires MD (NB : il ne s'agit pas d'un fit!). A droite : loi de diffusion intermédiaire calculée à partir de la trajectoire MD des centres de masse des molécules de iodométhane. Tiré de [31].

cristallins, ces matériaux présentent une conductivité thermique de type vitreuse. Des études combinant diffusion inélastique des neutrons, des rayons $\mathrm{X}$ et des calculs de dynamique de réseau ont permis d'expliquer ce comportement «anormal» à travers l'existence de fortes interactions entre les sousréseaux aqueux et invités. Soulignons que cet exemple met en jeu des processus de diffusion cohérente dans les clathrates hydrates. Le second exemple illustrant l'existence de fortes interactions hôte-invité concerne la diffusion incohérente inélastique des neutrons. L'étude des effets tunnel de rotation des molécules invitées a permis de mettre en évidence l'existence de sites d'adsorption à la surface des cages aqueuses d'un clathrate hydrate.

\subsection{Conductivité thermique}

Rappelons que la conductivité thermique d'un matériau est proportionnelle à sa capacité calorifique et au libre parcours moyen, $\lambda$, des phonons. Ce dernier est limité par deux phénomènes : la diffusion des phonons et la diffusion «géométrique ». Dans un cristal (voir le cas du quartz sur la partie droite de la Figure 7), la température diminuant, les collisions des phonons (processus Umklapp) deviennent moins fréquentes (se traduisant par une augmentation de $\lambda$ et, de ce fait, la conductivité thermique va augmenter pour atteindre un maximum lorsque $\lambda$ deviendra comparable à la dimension de l'échantillon (température de Debye). En dessous de cette température, les processus Umklapp ne limitent plus la conductivité thermique (diminution du nombre de phonon excités selon la loi de Boltzmann). L'effet de taille devient dominant si bien que la conductivité thermique varie comme la capacité calorifique (en $\mathrm{T}^{3}$ en dessous de la température de Debye). Dans le cas d'un verre (voir la silice vitreuse sur la Figure 7), la conductivité thermique est significativement plus faible que celle d'un cristal. Pour $\mathrm{T}<1 \mathrm{~K}$, la conductivité augmente suivant une loi en $\mathrm{T}^{2}$ comme attendu dans le cadre d'un modèle de Debye basé sur l'effet tunnel. Entre $1 \mathrm{~K}$ et $10 \mathrm{~K}$, il est observé un plateau pour lequel plusieurs interprétations sont proposées. Au dessus de $10 \mathrm{~K}$, la conductivité augmente faiblement pour tendre vers une valeur limite pouvant s'expliquer à partir des concepts de «modes mous » ou des phénomènes de diffusion résonnante entre les phonons acoustiques et des modes localisés.

Au cours de ces dernières décennies, les propriétés thermiques des clathrates hydrates ont fait l'objet de nombreuses études expérimentales et théoriques, eu égard au comportement «anormal»de leur conductivité thermique [32,33]. L'originalité de ce comportement tient dans deux aspects : 
(i) Bien que les clathrates hydrates soient des matériaux cristallins, la conductivité thermique présente un caractère «vitreux» (Figure 7). Cette observation a été faite sur plusieurs clathrates hydrates (voir les exemples cités dans la référence [34]).

(ii) Le sous-réseau hôte d'un clathrate hydrate présente une structure très proche de celle de la glace (réseau tétraédrique de molécule d'eau). La conductivité thermique est une des rares propriétés différant de manière drastique de celles de la glace [35].
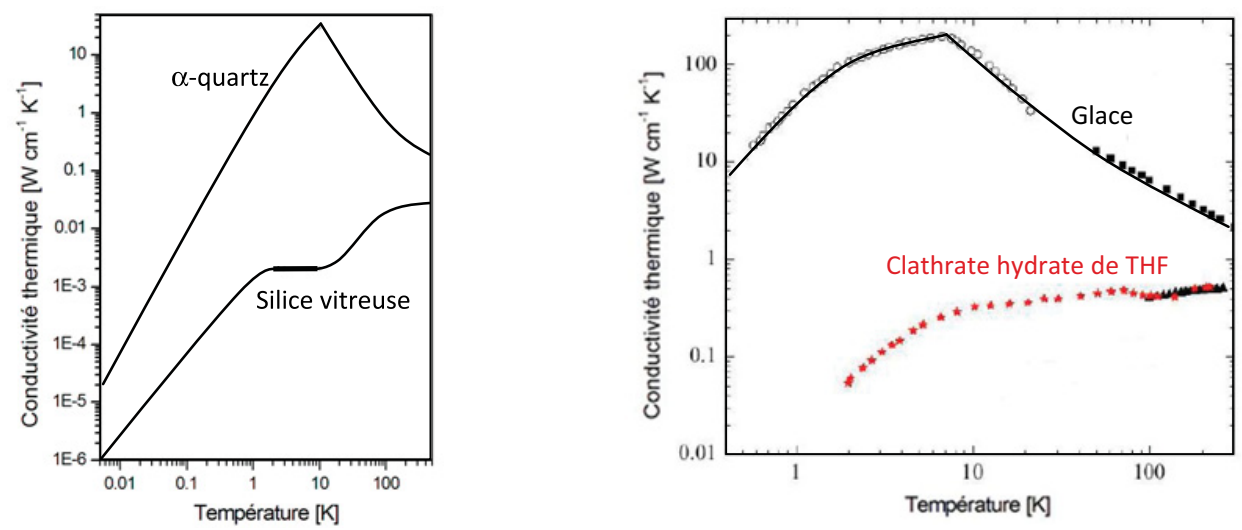

Figure 7. Dépendance en température de la conductivité thermique, à gauche, du quartz (cristallin) et de la silice vitreuse (d'après [36]) et à droite, du clathrate hydrate de tétrahydrofuranne (noté THF) et de la glace Ih (d'après [34]).

Vers la fin des années 80, deux hypothèses ont été émises quant à l'origine de ce comportement inhabituel : la taille des mailles élémentaires limitant le libre parcours moyen des phonons [37] ou un couplage entre modes de basse fréquence des sous-structures hôte et invitée influençant la diffusion des phonons [38]. Récemment, cette dernière hypothèse a été vérifiée par une série d'analyses expérimentales et théoriques [39]. Des calculs théoriques de dynamique de réseau ont mis en évidence un croisement évité entre les branches acoustiques du clathrate hydrate et les modes « rattling»des molécules incluses [40,41], comme illustré par les courbes de dispersion des phonons du clathrate hydrate de xénon (Figure 8). Ce phénomène est dû au fait que les branches des phonons mis en jeu possèdent les mêmes représentations irréductibles, autrement dit à l'existence d'interactions fortes entre les sous-structures aqueuses et invitées. Il est relativement difficile de confronter ces résultats théoriques à des expériences de diffusion inélastique des neutrons. En effet, de telles expériences doivent être effectuées sur un mono-cristal à l'aide d'un spectromètre 3-axes. Or, la cristallogenèse d'un monocristal de clathrates hydrates de taille suffisante est relativement complexe. Il est toutefois possible (comme illustré dans la partie 2) de comparer les modes « rattling » obtenus à partir de ces calculs de dynamique de réseau avec ceux obtenus en mesurant la densité d'état par diffusion inélastique des neutrons : comme représenté sur la Figure 8, un bon accord est observé, mais ce résultat ne permet pas de valider l'hypothèse d'un croisement évité.

Une solution expérimentale utilisée par J. Baumert et al consiste à mesurer les courbes de dispersion par diffusion inélastique des rayons X du clathrate hydrate de xénon et de méthane [42]. Cette technique permet d'atteindre des résolutions en énergie de l'ordre de $1.5 \mathrm{meV}$ et de sonder des transferts de moment compris entre $0.15 \AA^{-1}$ à $1.1 \AA^{-1}$. Il est alors possible d'analyser la dispersion des phonons dans la première zone de Brillouin d'un clathrate hydrate sous forme de poudre (partie gauche de la Figure 9). Rappelons que pour le clathrate de type I, la maille est cubique avec $2 \pi / \mathrm{a} \sim 0.5 \AA^{-1}$. A partir des courbes théoriques de dispersion, il est possible d'obtenir les spectres attendus de diffusion inélastique des rayons $\mathrm{X}$ en intégrant une moyenne de poudre dans ce calcul : ceux-ci reproduisent 

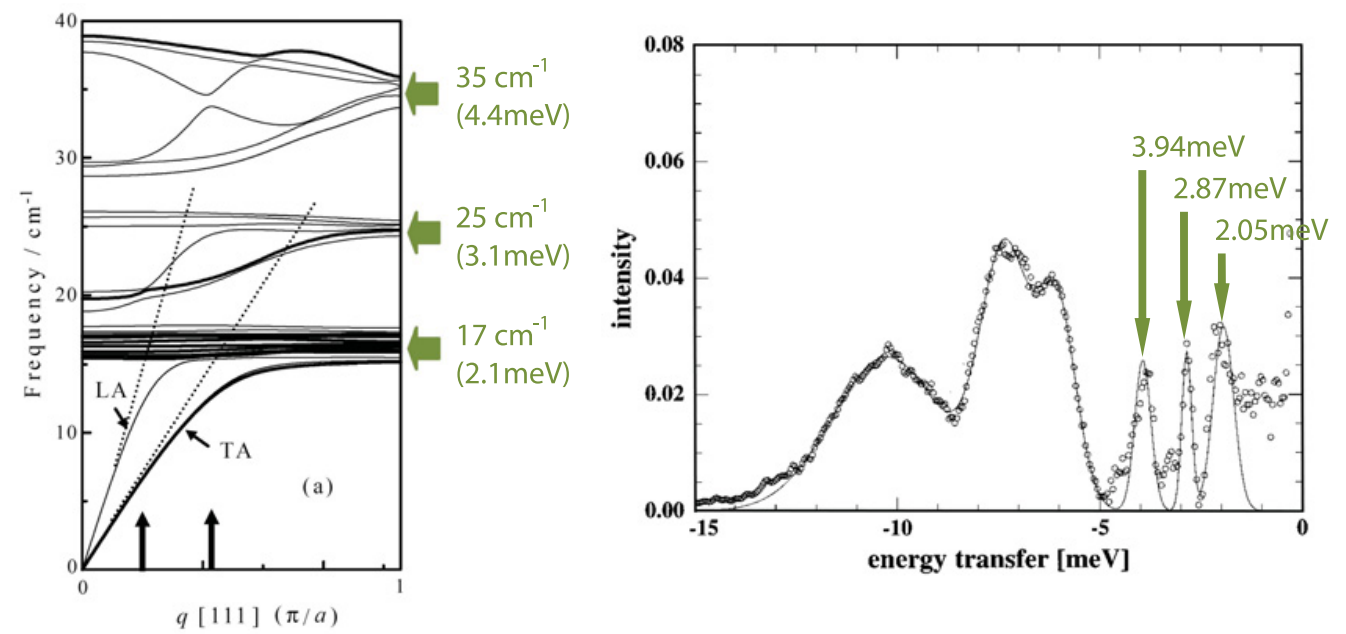

Figure 8. Courbes de dispersion calculées (à gauche) et densité d'états généralisée mesurée par diffusion inélastique des neutrons (à droite) du clathrate hydrate de xénon $\mathrm{Xe} \cdot 5.75 \mathrm{H}_{2} \mathrm{O}$ (type $\mathrm{I}$ ) à $\mathrm{T}=100 \mathrm{~K}$. Les énergies indiquées en vert correspondent au mode 《rattling» du xénon à $\sim 4 \mathrm{meV}$ dans les petites cages (de type $5^{12}$ ) et à deux composantes de ce mode localisé à $\sim 2 \mathrm{meV}$ et $\sim 3 \mathrm{mev}$ pour le xénon dans les grandes cages (de type $5^{12} 6^{2}$ ) du fait de la forme ellipsoïdale de ces cages. Tiré de [41,43].

les spectres expérimentaux du clathrate hydrate de méthane (Figure 9). En particulier, il est observé un transfert d'intensité des phonons acoustiques (perte) vers le mode «rattling»du méthane (gain) après le 《croisement» de ces deux branches (vers $0.2 \AA^{-1}$ ). Ces résultats prouvent ainsi l'existence d'un couplage des modes localisés des molécules invitées avec les phonons du sous-réseau aqueux, mécanisme de diffusion résonnante à l'origine de la faible conductivité thermique des clathrates hydrates.

\subsection{Sites d'adsorption}

D'une manière générale, les interactions hôte-invité peuvent être sondées par l'analyse de la dynamique rotationnelle des molécules invitées. Plus spécifiquement, l'obtention du potentiel de surface interne des cages aqueuses renseigne quant à l'existence de sites d'adsorption des molécules invitées à la surface des cages. A cet effet, des informations précises sont accessibles à travers l'analyse du tunneling rotationnel des molécules invitées. Pour que ce processus de dynamique quantique soit observable sur un spectre de diffusion inélastique des neutrons (méthode de choix pour ce type d'étude), les molécules doivent être faiblement «gênées » dans leurs réorientations (faible barrière énergétique du potentiel dans laquelle la molécule évolue). Tel est le cas des hydrates de méthane et d'halogénure de méthyle.

Dans cette section, nous ferons, dans un premier temps, un bref rappel sur l'effet tunnel de rotation et ses conséquences sur un spectre de diffusion inélastique des neutrons. Dans un second temps, nous détaillerons les résultats obtenus dans le cas du clathrate hydrate de iodo-méthane.

\subsubsection{Rappels sur l'effet tunnel de rotation}

L'effet tunnel de rotation permet de rendre compte des états librationnels d'une molécule dans laquelle les atomes en rotation sont indiscernables. Des groupements chimiques très faiblement gênés dans leur dynamique rotationnelle peuvent être le siège de transitions par effet tunnel. Le groupement méthyle, $\mathrm{CH}_{3}$, constitue certainement l'exemple le plus simple et le plus couramment rencontré de cette «dynamique quantique ». Sa dynamique rotationnelle par effet tunnel peut être décrite à l'aide d'un 

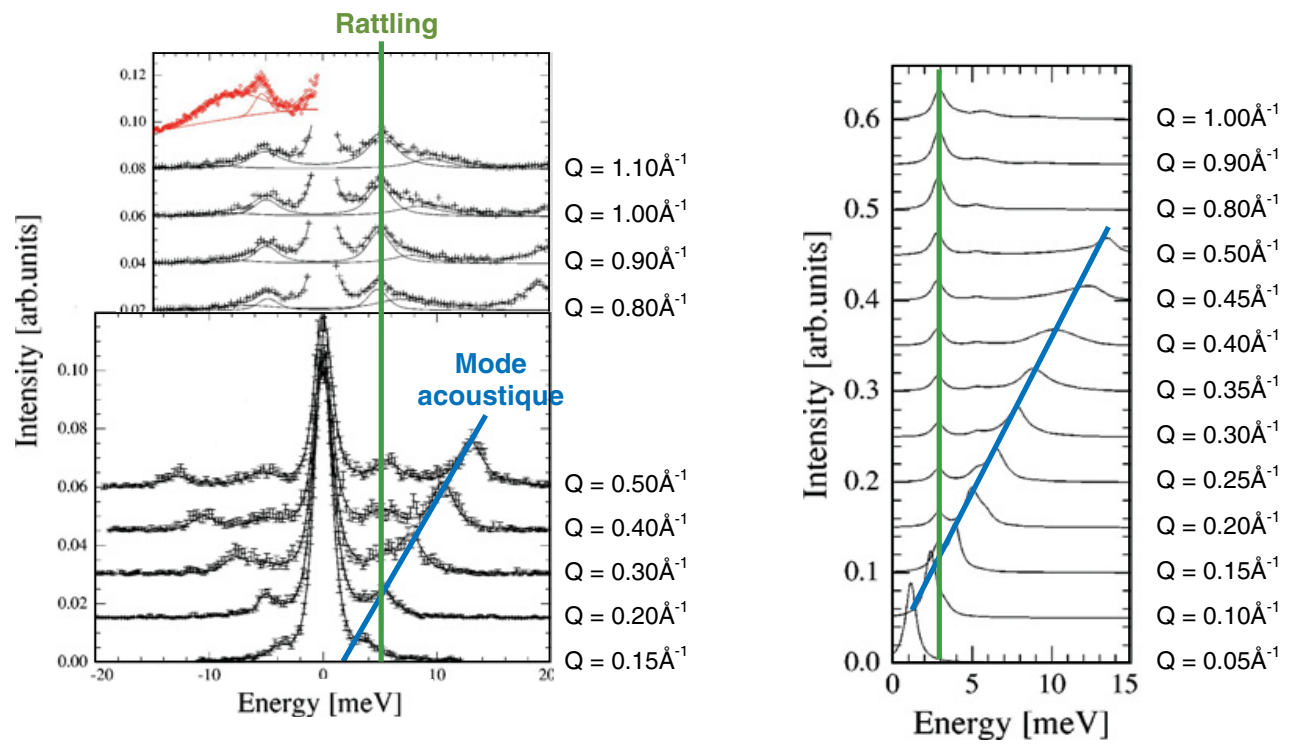

Figure 9. A gauche: Spectres de diffusion inélastique des rayons $\mathrm{X}$ dans la première zone de Brillouin du clathrate hydrate de methane $\left(\mathrm{CH}_{4}-\mathrm{H}_{2} \mathrm{O}\right)$ et de diffusion inélastique des neutrons (DOS du $\mathrm{CH}_{4}-\mathrm{D}_{2} \mathrm{O}$ en rouge). Le mode à $\sim 5 \mathrm{meV}$ est attribué au "rattling" des molécules de methane dans les grandes cages de la structure de type I (indiqué par une ligne verte). La ligne bleue correspond au mode longitudinal acoustique. A droite: Spectres théoriques de diffusion inélastique des rayons $\mathrm{X}$ calculés en considérant une moyenne de poudre du clathrate hydrate de méthane. Adapté de [42].

modèle de rotateur quantique unidimensionnel dans un potentiel caractéristique de son environnement. En se mettant dans l'approximation du rotateur rigide et en supposant que l'axe de rotation est fixe, ses niveaux d'énergie sont alors donnés par la résolution de l'équation de Schrödinger suivante [44,45] :

$$
\left[-B \frac{\partial^{2}}{\partial \varphi^{2}}+V(\varphi)\right] \Psi=E \Psi
$$

où $B$ correspond à la constante de rotation (pour le groupement méthyle $B=0.655 \mathrm{meV}$ ), $\varphi$ définit l'angle de rotation du groupement, $\Psi$ sont les fonctions d'onde du système et $E$ ses valeurs propres. Le potentiel de rotation $V(\varphi)$ peut être décrit par un développement de Fourier respectant la symétrie $C_{3}$ du méthyle :

$$
V(\varphi)=\sum_{n=1}^{\infty} V_{3 n}(1-\cos (3 n \varphi))
$$

Un développement au premier ordre du potentiel est, en général, suffisant pour interpréter les données de diffusion inélastique des neutrons. Une représentation de ce potentiel avec $n=1$ ainsi que des fonctions d'onde et valeurs propres associées est donnée sur la Figure 10. Chaque niveau librationnel $\left(E_{0}, E_{1} \ldots\right)$ est éclaté en deux sous-niveaux et de ce fait, deux types de transition peuvent donner lieu à des bandes sur un spectre de diffusion inélastique des neutrons. Des transitions entre niveaux librationnels «non éclatés $\gg E_{0} \rightarrow E_{1}$ seront observées à des énergies comprises entre $\sim 1 \mathrm{meV}$ et $\sim 300 \mathrm{meV}$ (modes de librations) et des transitions au sein de chacun des niveaux librationnels $E_{0}, E_{1} \ldots$, à des énergies de l'ordre du $\mu \mathrm{eV}$. Ces dernières transitions sont dues à l'effet tunnel et s'expliquent par l'existence d'un recouvrement des fonctions d'onde entre les puits de potentiel (la densité de probabilité de présence des atomes d'hydrogène est non nulle entre ces puits de potentiel). 

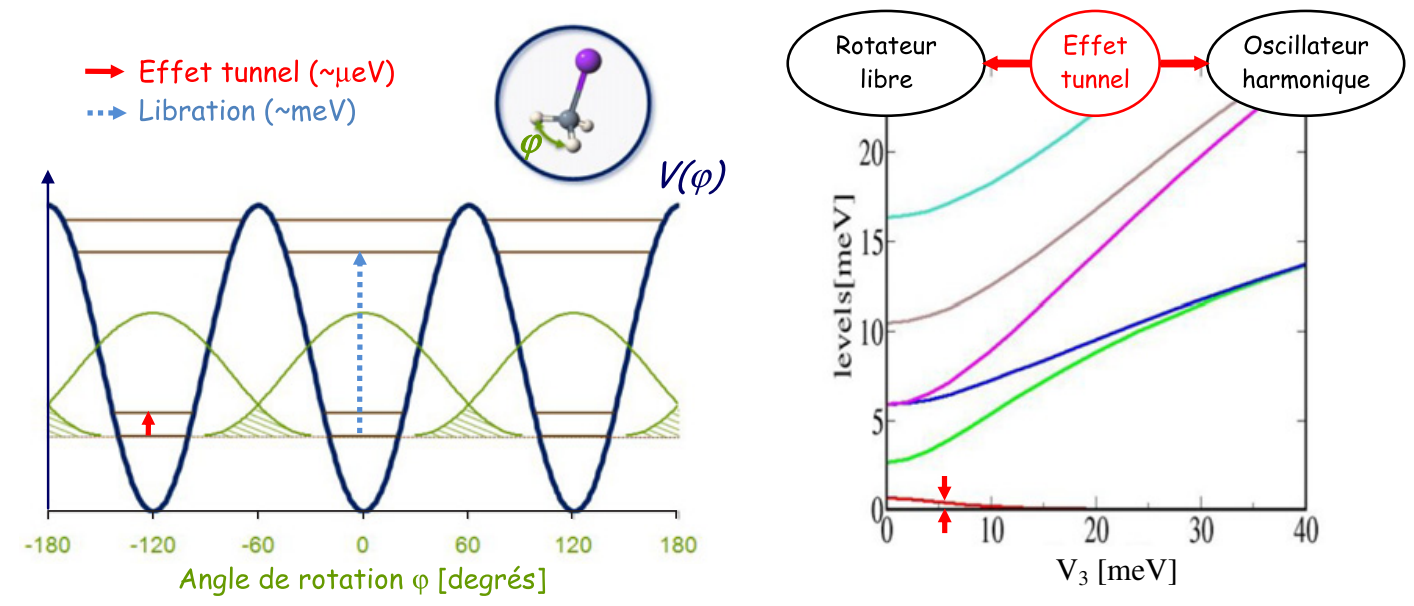

Figure 10. A gauche : représentation du potentiel $V(\varphi)$ (avec $n=1$ dans l'expression (2)) du groupement méthyle et des niveaux d'énergie de libration associés $\left(E_{0}, E_{1} \ldots\right)$. L'éclatement de ces niveaux est directement lié à la barrière de potentiel $V_{3}$. Les fonctions d'onde de l'état fondamental (et en particulier, leur recouvrement) sont également représentée en vert. Les unités utilisées sont arbitraires. A droite : Niveaux d'énergie de libration du groupement méthyle dans un potentiel $V(\varphi)$ (avec $n=1$ dans l'expression (2)) en fonction de la barrière d'énergie $V_{3}$.

L'éclatement par effet tunnel du niveau fondamental de libration, $E_{0}$, conduit à la loi de diffusion suivante $[44,45]$ :

$$
\begin{gathered}
S(Q, \omega)=A_{0}(Q) \delta(\omega)+\frac{1}{2}\left[1-A_{0}(Q)\right]\left[L\left(\omega+\omega_{t}\right)+L\left(\omega-\omega_{t}\right)\right] \\
\text { avec } A_{0}(Q)=\frac{5}{9}+\frac{4}{9} j_{0}(Q d) \quad \text { et } \quad L(\omega)=\frac{1}{\pi} \frac{\Gamma}{\Gamma^{2}+\omega^{2}}
\end{gathered}
$$

où $Q$ est le transfert de moment, $\hbar \omega_{t}$ correspond à l'éclatement par effet tunnel du premier niveau d'énergie librationnelle, $A_{0}(Q)$ est le facteur de structure élastique incohérent (EISF), $j_{0}(x)$ est la fonction de Bessel sphérique du premier ordre et $d$ est la distance entre deux protons au sein d'un groupement méthyle. La rotation par effet tunnel donnera ainsi naissance à deux pics inélastiques symétriques en énergie (voir l'exemple de la Figure 11) dont les intensités dépendront des populations relatives des niveaux librationnels initiaux et finaux, c'est-à-dire du facteur de Boltzmann, $\exp (\hbar \omega / \mathrm{kT})$. A une température donnée, les interactions du rotateur avec les phonons du système va induire une fluctuation dépendante du temps du potentiel qu'il faut introduire dans l'expression (1) (voir la référence [44] pour les détails du Hamiltonien). La conséquence de ces fluctuations sur les transitions par effet tunnel est un décalage vers les basses énergies et un élargissement des pics inélastiques lorsque la température augmente. L'élargissement $\Gamma$ suit un comportement de type Arrhénius [46] :

$$
\Gamma=\Gamma_{0} \exp \left(-\frac{E_{t}}{k T}\right)
$$

où $k$ est la constante de Boltzmann, $\Gamma_{0}$ est un préfacteur et $E_{t}$ représente l'énergie d'activation. Dans le cadre de la théorie des perturbations au second ordre [44], cet élargissement s'interprète comme le résultat d'un couplage résonnant avec les phonons du système à une énergie correspondant au premier niveau librationnel $E_{01}$ du rotateur isolé. Cette interprétation conduit alors à :

$$
E_{t}=E_{01}
$$


Un dernier point à souligner concerne l'inclusion des groupements méthyle dans les différents types de cage d'un clathrate hydrate. Les différentes structures des cages $\left(5^{12}, 5^{12} 6^{2}\right.$, etc...) va engendrer des rotateurs explorant différentes surfaces d'énergie potentielle. La conséquence est alors une distribution des pics inélastiques dus aux transitions par effet tunnel. Dans le cas d'un rotateur 1-D tel que le groupement méthyle, la loi de diffusion s'écrira :

$$
S(Q, \omega)=\sum_{k=1}^{N} p_{k} S_{k}(Q, \omega) \quad \text { avec } \quad \sum_{k=1}^{N} p_{k}=1
$$

où $p_{k}$ est la probabilité du rotateur $k$ et $S_{k}(Q, \omega)$ est la loi de diffusion correspondante, donnée par l'expression (3). Ainsi, le spectre de diffusion inélastique des neutrons sera composé de $N$ pics inélastiques associés aux $N$ rotateurs inéquivalents. Les facteurs de structure (expression (4)) étant identiques pour tous les rotateurs, les rapports d'intensité des différents pics (à condition de pouvoir les déconvoluer) permettra de remonter directement aux populations (probabilités) de chaque type de rotateur.

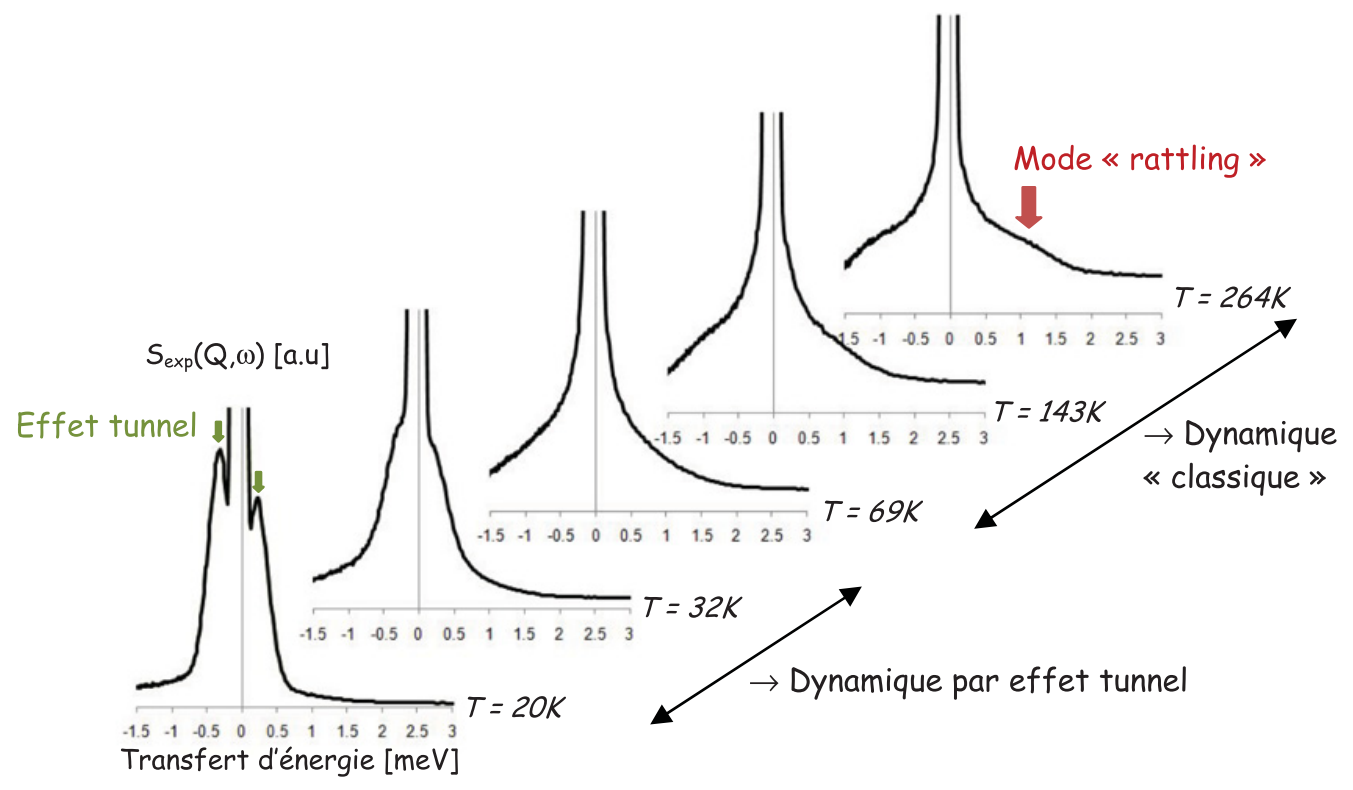

Figure 11. Spectres de diffusion incohérente quasi-(in)élastique des neutrons $\left(\lambda_{0}=5 \AA, \Delta \mathrm{E} \sim 100 \mu \mathrm{eV}\right.$ et $\langle\mathrm{Q}\rangle=$ $1.4 \AA^{-1}$ ) enregistrés sur un échantillon de clathrate hydrate de iodométhane avec le spectromètre temps de vol IN5 (ILL, Grenoble, France) [31].

Il est important de souligner les conditions dans lesquelles les transitions par effet tunnel peuvent être observées. Tout d'abord le potentiel du groupement méthyle doit présenter des barrières relativement faibles (de l'ordre de quelques meV). Autrement dit, la dynamique rotationnelle doit être «peu gênée ». Cet aspect est illustré sur la Figure 10, représentant les niveaux d'énergie de libration du groupement méthyle en fonction de la barrière d'énergie $V_{3}$ (voir l'expression (2)). Les deux cas limites à considérer sont les suivants : une barrière nulle correspondra au cas du rotateur libre et le cas de l'oscillateur harmonique sera rencontré pour des barrières élevées. Les transitions par effet tunnel seront observables pour des barrières comprises entre ces deux cas. De plus, étant généralement observées à faible transfert d'énergie $(\sim \mu \mathrm{eV})$, il est nécessaire d'utiliser des spectromètres à haute résolution en énergie (spectromètre à rétrodiffusion). Enfin, afin de s'affranchir de l'agitation thermique et de ne peupler que l'état librationnel fondamental, ces transitions ne sont observables qu'à basse température (de l'ordre 
de quelques K). Pour des températures supérieures à $100 \mathrm{~K}$ typiquement, cette dynamique rotationnelle quantique se transforme en mouvements stochastiques de réorientation pouvant être décrits dans le cadre de la mécanique classique. Expérimentalement, comme représenté sur la Figure 11, une augmentation de température se traduira par un élargissement des bandes inélastiques dues aux transitions par effet tunnel et un déplacement vers le pic élastique de celles-ci (apparition d'un spectre quasi-élastique).

Ainsi, l'étude des transitions d'effet tunnel par diffusion inélastique des neutrons constitue une sonde unique et très sensible du potentiel dans lequel une molécule évolue et donc des interactions spécifiques pouvant exister entre cette molécule et son environnement. Dans le cas des clathrates hydrates (pouvant être aisément généralisé à tous types de matériaux nanoporeux), le potentiel de surface interne des cages peut être précisément exploré à travers l'étude des transitions d'effet tunnel des groupements méthyle dans des clathrates hydrates d'halogénure de méthyle comme illustré dans la section suivante.

\subsubsection{Exemple de l'hydrate de iodo-méthane}

Du fait de sa simplicité et de sa symétrie moléculaire, le méthane est un rotateur tridimensionnel prototype. De plus, son premier moment multipolaire électrique non-nul est de nature octupolaire. Ainsi, la molécule de méthane dans les cages d'un hydrate représente un système modèle de molécule non-polaire. Toutefois, le caractère 3-D de ce rotateur rend l'analyse de l'effet tunnel par diffusion inélastique des neutrons [40-48] relativement complexe dans le cadre de ce cours. En effet, le clathrate hydrate de méthane cristallise dans une structure de type I dans laquelle les deux types de cages sont remplis. Un désordre statique des orientations des molécules au sein des cages couplé à un désordre du réseau de liaison hydrogène de la sous-structure hôte conduit à un potentiel caractérisé par une forte inhomogénéité des barrières. La distribution de ces barrières a pu être quantitativement analysée à l'aide de simulations de dynamique moléculaire [47]. De manière complémentaire, les clathrates hydrates d'halogénure de méthyle $\left(\mathrm{CH}_{3} \mathrm{X}\right.$ avec $\left.\mathrm{X}=\mathrm{I}, \mathrm{Cl}, \mathrm{F}, \mathrm{Br} \ldots\right)$ représentent des systèmes modèles de molécules polaires permettant de sonder les interactions dipôle-dipôle avec la surface des cages aqueuses. De plus, ces molécules invitées possèdent un axe de symétrie $\mathrm{C}_{3}$ (la liaison $\mathrm{C}-\mathrm{X}$ ), leur conférant un caractère rotationnel 1-D et rendant la description mathématique (voir section précédente) beaucoup plus simple que celle du méthane (3-D). L'étude de la dynamique rotationnelle par effet tunnel est disponible dans la littérature pour des structures de type I formées avec les molécules de bromo-méthane [49], de fluoro-méthane [50] et de chloro-méthane [51]. Comme dans le cas du méthane, l'analyse des spectres est rendue complexe par le fait que les deux types de cages de la structure de type I sont remplies. D'un point de vue pédagogique, le clathrate hydrate de iodo-méthane est plus simple. En effet, les molécules encapsulées sont a priori toutes dans un même environnement puisque celles-ci n'occupent que les grandes cages (de type $5^{12} 6^{4}$ ) de la structure de type II formée. Ce système constitue ainsi un très bon système modèle pour sonder les interactions hôtes-invitées par spectroscopie d'effet tunnel (diffusion inélastique des neutrons). Des informations précises quant aux sites d'adsorption à la surface des cages aqueuses peuvent ainsi être obtenues [52,53], comme nous allons le décrire.

Le spectre de diffusion inélastique des neutrons du clathrate hydrate de iodo-méthane, $\mathrm{CH}_{3} \mathrm{I} \times$ $17 \mathrm{D}_{2} \mathrm{O}$, est présenté sur la Figure 12. Trois points sont à souligner. Tout d'abord, plusieurs transitions par effet tunnel sont observées sur ce spectre alors qu'a priori, une seule bande est attendue (toutes les molécules de iodométhane sont cristallographiquement équivalentes). La deuxième observation concerne l'évolution temporelle du spectre (différence entre les spectres $1 \mathrm{~h}$ et $5 \mathrm{~h}$ après avoir atteint la température de mesure $1.8 \mathrm{~K}$ sur la Figure 12). Cette différence est due au fait que le système se trouve hors d'équilibre thermique sur l'échelle de temps d'acquisition d'un spectre de diffusion inélastique des neutrons (de l'ordre de la dizaine d'heure). En effet, l'état librationnel fondamental du groupement méthyle possède un spin 3/2 alors que le spin du premier état excité est de 1/2: la transition par effet tunnel implique donc une conversion de spin caractérisée par une constante de temps de l'ordre de plusieurs dizaines d'heure dans des matériaux moléculaires tels que les clathrates hydrates [44,52]. La dernière observation concerne l'asymétrie au niveau de la forme des bandes entre les parties gain 


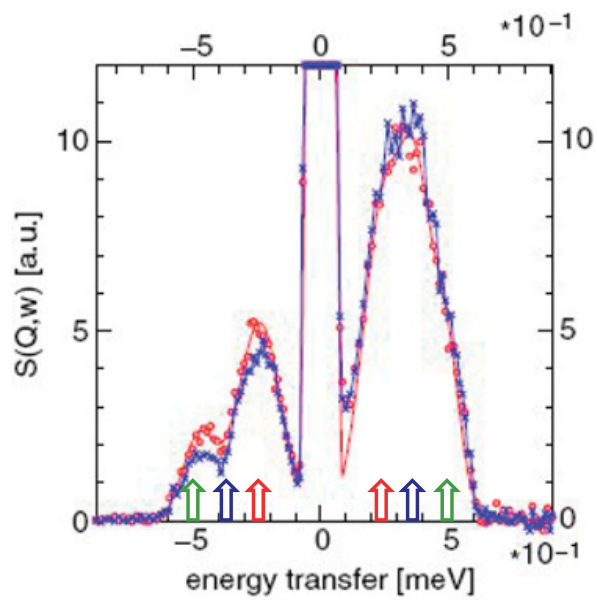

Trois transitions par effet tunnel:

\begin{tabular}{|c|c|c|}
\hline $\mathrm{h} \omega_{+}[\mu \mathrm{eV}]$ & $\Gamma[\mu \mathrm{eV}]$ & $p_{k}$ \\
\hline$\Rightarrow 242$ & 90 & 0.53 \\
\hline$\Rightarrow 372$ & 80 & 0.31 \\
\hline 500 & 65 & 0.16 \\
\hline
\end{tabular}

Figure 12. Spectres de diffusion inélastique des neutrons enregistrés sur un échantillon de clathrate hydrate de iodo-méthane $\mathrm{CH}_{3} \mathrm{I} \cdot 17 \mathrm{H}_{2} \mathrm{O}$ avec le spectromètre temps de vol NEAT (HMI, Berlin). $\lambda_{0}=7.0 \AA, \Delta \mathrm{E}_{\text {res }}=50 \mu \mathrm{eV}$, $\mathrm{Q} \sim 1.6 \AA^{-1}$. Points $(\mathrm{\circ})$ : $1 \mathrm{~h}$ après avoir atteint la température de $1.8 \mathrm{~K}$; croix $(\times)$, après $5 \mathrm{~h}$. La ligne continue rouge correspond au fit des données expérimentales. Les flèches verte, bleue et rouge correspondent aux transitions par effet tunnel et le tableau de droite donne la position $\hbar \omega_{t}$, la largeur $\Gamma$ et l'intensité relative $\mathrm{p}_{k}$ (des expressions (3) et (7)) de chacun des pics. Tiré de [52].

d'énergie et perte d'énergie. Deux bandes peuvent être observées du coté gain d'énergie $(\hbar \omega<0)$. En utilisant les positions et largeurs de ces deux bandes et un rapport d'intensité suivant la loi de Boltzmann, il n'est pas possible de reproduire le spectre du coté gain d'énergie $(\hbar \omega>0)$. Il est indispensable d'ajouter une troisième bande pour reproduire l'intégralité du spectre. L'ajustement du spectre a donc été réalisé à l'aide des équations (3) et (7) avec $N=3$. Les largeurs, positions et populations relatives des trois types de rotateurs sont donnés sur la Figure 12. Il faut noter que chaque cage d'un même type $\left(5^{12} 6^{4}\right)$, bien qu'identique en termes de position des atomes d'oxygène, peut présenter une distribution différente des liaisons hydrogène. Ces inhomogénéités vont engendrer des rotateurs équivalents mais se réorientant dans des potentiels légèrement différents, et ainsi être à l'origine des largeurs des bandes inélastiques. Enfin, le résultat de l'ajustement présenté en ligne continue sur la Figure 12 intègre la relation suivante pour rendre compte du rapport d'intensité des bandes pour $\hbar \omega<0$ (noté $I_{-}$) et pour $\hbar \omega>0$ (noté $\left.I_{+}\right)$:

$$
\frac{I_{-}}{I_{+}}=\exp \left(-\frac{\hbar \omega_{t}}{k T_{\text {spin }}}\right)
$$

où $T_{\text {spin }}$ représente la température de spin du rotateur considéré. Pour les trois transitions, ces températures sont supérieures à celle de l'échantillon : pour les spectres enregistrés après 1 heure à $\mathrm{T}=1.8 \mathrm{~K}, T_{\text {spin }}=14.5 \mathrm{~K}, 5.1 \mathrm{~K}$ et $14.1 \mathrm{~K}$ pour les bandes à $\hbar \omega_{t}=500 \mu \mathrm{eV}, 372 \mu \mathrm{eV}$ et $242 \mu \mathrm{eV}$, respectivement. Les phénomènes de conversion de spin sont à l'origine des différences de température observées. Chacun des trois types de rotateur possède son propre temps caractéristique de conversion de spin [52]. Autrement dit, l'interaction avec la sous-structure aqueuse diffère d'un rotateur à l'autre. Ce résultat est surprenant étant donné que chaque molécule de iodométhane est cristallographiquement équivalente : elles sont toutes encapsulées dans des cages de type $5^{12} 6^{4}$. Une explication plausible pour ces différences d'interaction réside dans l'existence de différents sites d'adsorption à la surface des cages. Trois origines structurales sont possibles: les molécules de iodométhane pointent (i) vers les atomes d'oxygène, (ii) vers le centre des polygones formant les cages ou (iii) vers les arêtes de 
ces polygones (contenant les liaisons hydrogène). L'hypothèse (i) peut être exclue étant donné que seulement deux types d'atome d'oxygène cristallographiquement différents forment les cages de type $5^{12} 6^{4}$ alors que trois transitions par effet tunnel sont observées. De même, l'hypothèse (ii) peut être exclue puisque les cages sont formées par des pentagones et des hexagones. La dernière hypothèse (iii) semble donc la plus plausible, et de plus, reproduit les rapports d'intensité observés pour les transitions par effet tunnel. En effet, une cage de type $5^{12} 6^{4}$ est formée par 42 liaisons hydrogène (42 arêtes) dont 57\% d'entre elles relie deux pentagones, $14 \%$ relie deux hexagones et $29 \%$ relie un hexagone et un pentagone (voir Figure 13). Ces populations reproduisent correctement les rapports d'intensité associés aux trois types de rotateurs. Ainsi, les trois sites d'adsorption déterminés par l'analyse des transitions par effet tunnel peuvent être associés à l'existence de trois «types » de liaison hydrogène au sein de la sous-structure aqueuse.
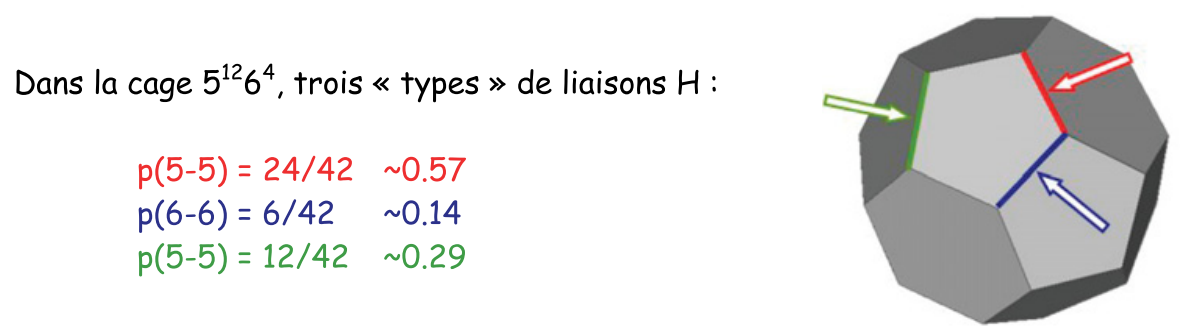

Figure 13. Représentation des trois «types » de liaisons hydrogène existant au sein d'une cage de type $5^{12} 6^{4}$ et de leur population relative.

\section{CONCLUSION}

Les études menées sur les clathrates hydrates sont actuellement en plein essor du fait, en particulier, des nombreuses implications dans le domaine énergétique. Ces développements finalisés reposent sur une compréhension, à un niveau fondamental, des mécanismes microscopiques régissant les propriétés spécifiques de ces matériaux. Ce point a été illustré à travers plusieurs exemples parmi lesquels le comportement anormal (de type «vitreux») de la conductivité thermique des clathrates hydrates (matériaux cristallins) est certainement le plus marquant. De plus, la structuration particulière des clathrates (sous forme de cages encapsulant des molécules) leur confèrent des propriétés fondamentalement originales. L'ensemble des excitations rencontrées dans ces solides a été exposé pour des énergies allant de quelques $\mu \mathrm{eV}$ à quelques centaines de meV. En particulier, les modes «hochet» (ou «rattling») des molécules encapsulées constituent des signatures spectroscopiques à basse fréquence des clathrates hydrates.

D'un point de vue expérimental, l'apport de la diffusion inélastique des neutrons est significatif dans l'étude de la dynamique de ces matériaux, autrement dit, afin de mieux comprendre les interactions hôte-invité rencontrées. Par exemple, l'analyse du tunneling rotationnel des molécules encapsulées permet d'explorer le potentiel de surface interne des cages aqueuses. De telles études ont permis de mettre en évidence l'existence de sites d'adsorption des molécules invitées à la surface des cages. Une limite de la diffusion inélastique des neutrons dans l'étude de ces matériaux réside dans la difficulté de préparation de monocristaux. De ce fait, la combinaison de plusieurs techniques spectroscopiques de diffusion (Raman, rayons X...) est souvent indispensable pour explorer les interactions hôteinvitée de ces systèmes. D'un point de vue méthodologique, l'apport des simulations numériques (dynamique de réseau, dynamique moléculaire...) est indispensable pour guider l'interprétation des résultats expérimentaux. 


\section{Remerciements}

L'élaboration de ce cours repose, en partie, sur les recherches que j'effectue sur les clathrates hydrates avec J.C. Soetens (ISM, Talence), M. Russina, E. Kemner (HZB, Berlin), J. Ollivier, M.A. Gonzalez (ILL, Grenoble), E. Pefoute et F. Mançois (doctorants ISM), que je tiens à remercier. A travers cet article, je souhaite également rendre un hommage particulier à M. Prager.

\section{Références}

[1] G. A. Jeffrey, in Comprehensive Supramolecular Chemistry, Hydrate Inclusion Compounds, edited by J. L. Atwood, J. E. D. Davies, D. D. Mac-Nicol, and F. Vögtle (Pergamon, Oxford) Vol. 6, p. 757 (1996)

[2] E.D. Sloan, in Clathrate Hydrates of Natural Gases, NewYork: Marcel Dekker (1998)

[3] F. Hollander et G.A. Jeffrey, J. Chem. Phys. 66, 4699 (1977)

[4] C. Gutt, B. Asmussen, W. Press, M.R. Johnson, Y.P. Handa et J. Tse J. Chem. Phys. 113, 4713 (2000)

[5] C.A. Koh, E.D. Sloan, AIChE Journal, 53(7), 1636 (2007)

[6] I. Chatti, A. Delahaye, L. Fournaison, J.P. Petitet, Energy Conversion and Management 46, 1333 (2005)

[7] E.D. Sloan, Nature 426, 353 (2003)

[8] M. Nakasako, J. Biol. Phys. 28, 129 (2002)

[9] H. Lee, J.-W. Lee, D.Y. Kim, J. Park, Y.T. Seo, H. Zeng, I.L. Moudrakovski, C.I. Ratcliffe and J. Ripmeester, Nature, 434, 743 (2005)

[10] L.J. Florusse, C.J. Peters, J. Schoonman, K.C. Hester, C.A. Koh, S.F. Dec, K.N. Marsh, E.D. Sloan, Science 306, 469 (2004)

[11] W.L. Mao, H.K. Mao, A.F. Goncharov, V.V. Struzhkin, Q.Z. Guo, J.Z. Hu, J.F. Shu, R.J. Hemley, M. Somayazulu, Y.S. Zhao, Science 2972247 (2002)

[12] C.A. Koh, Chem. Soc. Rev. 31157 (2002)

[13] H. Schober, EDP Sciences, collection SFN 10 (2010)

[14] H. Schober, J. Phys. IV France 103, 173 (2003)

[15] M. Bée, J. Phys. IV France 111259 (2003)

[16] M. Jonhson, EDP Sciences, collection SFN 10 (2010)

[17] M. Bach-Verge's, S. J. Kitchin, K. D. M. Harris, M. Zugic, C. A. Koh, J. Phys. Chem. 105, 2699 (2001)

[18] T. M. Kirschgen, M. D. Zeidler, B. Geil and F. Fujara, Phys. Chem. Chem. Phys. 5, 5243 (2003)

[19] T. M. Kirschgen, M. D. Zeidler, B. Geil and F. Fujara, Phys. Chem. Chem. Phys. 5, 5247 (2003)

[20] A. Desmedt, F. Stallmach, R.E. Lechner, D. Cavagnat, J.C. Lassègues, F. Guillaume, J. Grondin, M.A. Gonzalez, J. Chem. Phys. 121(23), 11916 (2004)

[21] F. Guillaume, M. Couzi, EDP Sciences, collection SFN 10 (2010)

[22] I.M. Chou, A. Sharma, R.C. Burruss, J. Shu, H. Mao, R.J. Hemley, A.F. Goncharov, L.A. Stern, S.H. Kirby, PNAS 97, 13485 (2000)

[23] H. Schober, H. Itoh, A. Klapproth, V. Chihaia, W.F. Kuhs, Eur. Phys. J. E 12, 41 (2003)

[24] J.S. Tse, D.D. Klug, in Proceedings of the Fourth International Conference on Gas Hydrates, Yokohama, 669 (2002)

[25] S. Subramanian, E.D. Sloan, Fluid Phase Equilibria 158-160, 813 (1999)

[26] E.D. Sloan, J. Chem. Thermodynamics 35, 41 (2003)

[27] J. P. Ledheros et al, Chem. Eng. Sci. 51(8), 1221 (1996)

[28] B. Chazallon, H. Itoh, M. Koza, W. F. Kuhs and H. Schober, Phys. Chem. Chem. Phys. 4, 4809 (2002) 
[29] J. S. Tse, V. P. Shpakov, V. R. Belosludov, F. Trouw, Y. P. Handa et W. Press, Europhys. Lett. 54, $354(2001)$

[30] H. Mutka, EDP Sciences, collection SFN 9 (2008)

[31] A. Desmedt, J.C. Soetens, F. Mançois, M. Prager, M. Russina, J. Ollivier, article en préparation.

[32] R.D. Stoll, G.M. Bryan, J. Geophys. Res. 84, 1629 (1979)

[33] R.G. Ross, P. Andersson, G. Backstrom, Nature 290, 322 (1981)

[34] J. Baumert, Thèse de l'université de Kiel (2003)

[35] Y. P. Handa, J.G. Cook, J. Phys. Chem. 91(25), 6321 (1987)

[36] R.C. Zeller, R.O. Pohl, Phys. Rev. B 4(6), 2029 (1971)

[37] M.W.C. Dharma-Wardana, J. Phys. Chem. 87, 4185 (1983)

[38] J.S. Tse, M.A. White, J. Phys. Chem. 92, 5006 (1988)

[39] J.S. Tse, D.D. Klug, J.Y. Zhao, W. Sturhahn, E.E. Alp, J. Baumert, C. Gutt, M.R. Johnson et W. Press, Nature Materials 4, 917 (2005)

[40] J.S. Tse, C.I. Ratcliffe, B.M. Powell, V.F. Sears, Y.P. Handa, J. Phys. Chem., 101, 4491 (1997)

[41] J. S. Tse, V. P. Shpakov, V. R. Belosludov, F. Trouw, Y. P. Handa, W. Press, Europhys. Lett. 54, 354 (2001)

[42] J. Baumert, C. Gutt, V. P. Shpakov, J. S. Tse, M. Krisch, M. Müller, H. Requardt, D. D. Klug, S. Janssen, W. Press, J. Chem. Phys. 116(9), 3795 (2002)

[43] C. Gutt, J. Baumert, W. Press, J.S. Tse, S. Janssen, J. Chem. Phys. 116(9), 3795 (2002)

[44] M. Prager, A. Heidemann, Chem. Rev 97, 2933 (1997)

[45] W. Press et al, "Single-particle rotations in molecular crystals", Springer Tracts in Modern Physics 92, Springer-Berlin (1981)

[46] A.C. Hewson, J. Phys. C: Solid State Phys. 15, 3841 et 3855 (1982)

[47] C. Gutt, W. Press, A. Hüller, J.S. Tse, H. Casalta, J. Chem Phys. 114(9), 4160 (2001)

[48] C. Gutt, W. Press, A. Hüller, J.S. Tse Appl. Phys. A 74 [Suppl.], S1299-S1301 (2002)

[49] M. Prager, A. Desmedt, J. Allgaier, M. Russina E. Jansen, I. Natkaniec, A. Paluwkojc, W. Press Phase Transitions 80(6-7) 473 (2007)

[50] M. Prager, J. Baumert, W. Press, M. Plazanet, J. S. Tse, D. D. Klug, Phys . Chem. Chem. Phys. 7, $1228(2005)$

[51] M. Prager, A. Desmedt, T. Unruh, J. Allgaier J. Phys.: Condens. Matter 20, 125219 (2008)

[52] M. Prager, J Pieper, A. Buchsteiner, A. Desmedt, J. Phys.: Condens. Matter 167045 (2004)

[53] M. Prager, J. Pieper, A. Buchsteiner, A. Desmedt Physica B 350, e399 (2004) 